\title{
Uncertainty Evaluation in Determination of Boron and Lead in Plastic by ICP-AES
}

\author{
Meng $\mathrm{Li}^{1 \dagger}$, Huanyong $\mathrm{Li}^{2, \dagger *}$, Zepeng $\mathrm{Jiao}^{2}$, Lin Wang ${ }^{2}$, Lvhuan Wang ${ }^{2}$ \\ ${ }^{1}$ College of Life Science and Technology, Jinan University, Guangzhou 510632, China \\ ${ }^{2}$ Analytical and Testing Center, Jinan University, Guangzhou 510632, China \\ *Corresponding email: tlihuanyong@jnu.edu.cn \\ $\dagger$ these authors contributed equally to this work
}

Keywords: Plastic, Toxic trace element, Microwave digestion, ICP-AES, Uncertainty.

Abstract: The content of toxic trace elements is an important index to measure the quality of plastic products. For a long time, the determination of toxic trace elements is the focus of third party detection and so on. It is very important to evaluate the accuracy of detection. In this paper, a mathematical model of the uncertainty of trace elements based on regression equation, constant volume and weighing weight is established. The results show that the content of Boron and Lead in the plastic samples is $(66.3 \pm 5.4) \mathrm{mg} / \mathrm{kg}$ and (500 $\pm 30) \mathrm{mg} / \mathrm{kg}$, respectively.

\section{Introduction}

Plastic pollution is one of the environmental issues of common concern around word [1-3]. The United Nations Environment Programme (UNEP) 2018 World Environment Day[4] is the theme "Beat Plastic Pollution", calling for concerted efforts by all countries in the world to combat the problem of plastic pollution. In recent years, the research of plastic testing in China has developed rapidly[5,6]. Some progress has been made in the aspects of the monitoring and analysis methods of plastics, the pollution characteristics and distribution of pollutants in the environment, the environmental process and behavior , the accumulation and the toxic effects of plastics. The content of toxic trace elements is an important index to measure the quality of plastic products. For a long time, the determination of toxic trace elements is the focus of third- party detection institution and so on. It is very important to evaluate the accuracy of detection. In this paper, a mathematical model of the uncertainty[7,8] of trace elements is established by evaluating the uncertainty of the determination results, which provides an effective method for evaluating the content of trace elements in the future. 


\section{Methods and results:}

\subsection{Measurement methods}

$0.2000 \mathrm{~g}$ plastic sample were used in the digestion tank for three times, and nitric acid was decomposed into a 50ml volumetric flask. The concentration of the element was measured by ICP-OES for three times.

$0,0.10,0.20,0.30,0.40,0.50 \mathrm{~mL}$ standard reserve solution(1000 $\mathrm{mg} / \mathrm{L})$ was removed by $1 \mathrm{~mL}$ pipette respectively,and then transfer the standard solution of different quantity in the $100 \mathrm{~mL}$ volumetric flask with the scale pipette and dilute it to the scale with water.The intensity of the calibration solution is measured under the same conditions , each solution is measured for three times, and the calibration curve is drawn using the average value.

\subsection{Results}

Table 1. The content of the element $\mathrm{B}$ and $\mathrm{Pb}$

\begin{tabular}{ccc}
\hline Element & $\mathrm{c}(\mathrm{mg} / \mathrm{L})$ & $\omega(\mathrm{mg} / \mathrm{kg})$ \\
\hline B & 0.2644 & 61.2 \\
$\mathrm{~B}$ & 0.2662 & 66.7 \\
$\mathrm{~B}$ & 0.2666 & 71.1 \\
$\mathrm{~Pb}$ & 2.086 & 535 \\
$\mathrm{~Pb}$ & 1.959 & 460 \\
$\mathrm{~Pb}$ & 2.040 & 505 \\
\hline
\end{tabular}

\section{Mathematical model of uncertainty}

Calculation formula of element content is:

$$
\omega=\frac{\mathrm{cV}}{\mathrm{m}}
$$

$\omega(\mathrm{mg} / \mathrm{kg}), \mathrm{c}(\mathrm{mg} / \mathrm{L}), \mathrm{V}(\mathrm{mL}), \mathrm{m}$ : The quality of the sample(g).

\subsection{Source of uncertainty}

The sources of uncertainty by the mathematical model include:

1.Uncertainty in the concentration of elements in a sample solution:u(c);

2.Uncertainty in the concentration of elements in a sample solution:u(V);

3.Uncertainty of the quality of the sample:u(m);

4.Uncertainty of repeated experiments:u(rep). 


\section{Evaluation of the relative standard uncertainty components}

\subsection{Evaluation of relative standard uncertainty of concentration in solution: $u_{\text {rel }}(c)$}

\subsubsection{Relative standard uncertainty of linear fitting of calibration curve: $u_{\text {rel }}(c)_{1}$}

From calibration curve $\mathrm{I}=\mathrm{a}+\mathrm{bc}$, we can see:

$$
\begin{gathered}
\mathrm{b}=\frac{\sum_{\mathrm{i}=1}^{\mathrm{n}}\left(\mathrm{c}_{\mathrm{i}}-\overline{\mathrm{c}}\right)\left(\mathrm{I}_{\mathrm{i}}-\overline{\mathrm{I}}\right)}{\sum_{\mathrm{i}=1}^{\mathrm{n}}\left(\mathrm{c}_{\mathrm{i}}-\overline{\mathrm{c}}\right)^{2}} \\
\mathrm{a}=\overline{\mathrm{I}}-\mathrm{b} \overline{\mathrm{c}}
\end{gathered}
$$

So, uncertainty of element concentration in solution from Calibration curve linear fitting $u(c)_{1}$ is

$$
\mathrm{u}(\mathrm{c})_{1}=\frac{\mathrm{s}_{\mathrm{R}}}{\mathrm{b}} \sqrt{\frac{1}{\mathrm{~m}}+\frac{1}{\mathrm{n}}+\frac{\left(\mathrm{c}_{0}-\overline{\mathrm{c}}\right)^{2}}{\sum_{\mathrm{i}=1}^{\mathrm{n}}\left(\mathrm{c}_{\mathrm{i}}-\overline{\mathrm{c}}\right)^{2}}}
$$

$\mathrm{S}_{\mathrm{R}}$ means standard deviation of calibrated solution:

$$
\mathrm{S}_{\mathrm{R}}=\sqrt{\frac{\left.\sum_{\mathrm{i}=1}^{\mathrm{n}}\left(\mathrm{I}_{\mathrm{i}}-=1 \mathrm{c}_{\mathrm{i}}+\mathrm{a}\right)\right)^{2}}{\mathrm{n}-2}}(\mathrm{~m}=9, \mathrm{n}=18)
$$

$\mathrm{u}(\mathrm{c})_{1}$ of element B and $\mathrm{Pb}$ was 0.0008 and $0.0004 \mathrm{mg} / \mathrm{L}, \mathrm{u}_{\mathrm{rel}}(\mathrm{c})_{1}$ was 0.004 and 0.002 , respectively.

\subsubsection{Uncertainty of standard solution concentration: $u_{\text {rel }}(c)_{2}$}

The standard solution concentration was $1000 \mathrm{mg} / \mathrm{L}, \mathrm{k}=2$, so the standard uncertainty: $\mathrm{u}_{\text {rel }}(\mathrm{c})_{2}=2$ $\mathrm{mg} / \mathrm{L}$, relative standard uncertainty: $u_{r e l}(c)_{2}=0.002$.

\subsubsection{Uncertainty of removing the volume of standard solution: $u_{\text {rel }}(c)_{3}$}

we removed $0,0.10,0.20,0.30,0.40,0.50 \mathrm{~mL}$ standard reserve solution of element B by using $1 \mathrm{~mL}$ pipette respectively to build calibration curve, According to GB/T $12805, \mathrm{u}_{\text {rel }}(\mathrm{c})_{3}$ of element B $=0.005$. similar to element $\mathrm{B}, \mathrm{u}_{\mathrm{rel}}(\mathrm{c})_{3}$ of element $\mathrm{Pb}$ is 0.005 .

\subsubsection{Uncertainty of instrument variability}

Since the repeatability of the measurement method has been calculated, the variability of the instrument has been included and is no longer calculated.

4.1.5 Relative Standard uncertainty of element concentration in sample solution:

$$
\mathrm{u}_{\mathrm{rel}}(\mathrm{c})=\sqrt{\mathrm{u}_{\mathrm{rel}}^{2}(\mathrm{c})_{1}+\mathrm{u}_{\mathrm{rel}}^{2}(\mathrm{c})_{2}+\mathrm{u}_{\mathrm{rel}}^{2}(\mathrm{c})_{3}}
$$

So, $\mathrm{u}_{\text {rel }}(\mathrm{c})$ of element $\mathrm{B}$ and $\mathrm{Pb}$ is 0.007 and 0.05 , respectively. 


\subsection{Evaluation of relative standard uncertainty of constant volume of sample solution: $u_{\text {rel }}(V)$}

The test solution was diluted with water in a $50 \mathrm{ml}$ volumetric flask. According to GB/T 12806, tolerance of $50 \mathrm{~mL}$ volumetric flask in class $A$ is $\pm 0.5 \mathrm{~mL} . \mathrm{u}(\mathrm{V})=0.02 \mathrm{ml} . \mathrm{u}_{\text {rel }}(\mathrm{V})=0.0004$.

If the sample is repeatedly measured several times, usually several $50 \mathrm{ml}$ volumetric flasks are used. It can be considered that the volume error and reading repeatability have been randomized, and the volume uncertainty component can be ignored.

\subsection{Evalution of relative standard uncertainty resulted from weighing sample quality by balance: $\mathbf{u}_{\text {rel }}(\mathbf{m})$}

The quality weighed of the samples is $0.2000 \mathrm{~g}$. According to the certificate, the maximum allowable difference of the balance is $\pm 0.5 \mathrm{mg}$, weigh twice, once empty, once with plate, and evenly distributed, $\mathrm{k}=\sqrt{3}$.

The standard uncertainty of the sample quality weighed $: u(m)=\sqrt{2 \times(0.5 / \sqrt{3})^{2}}=0.41 \mathrm{mg}$.

Relative standard uncertainty: $u_{\text {rel }}(m)=0.41 / 200=0.002$

\section{4 evaluation of relative standard uncertainty introduced by repetitive experiments : $\mathbf{u}_{\text {rel }}(\mathbf{r e p})$}

The standard uncertainty introduced by repetitive experiments is

$\mathrm{u}_{\mathrm{rel}}(\mathrm{rep})$ is both 0.03 .

$$
\mathrm{u}(\mathrm{rep})=\mathrm{s}(\omega) / \sqrt{\mathrm{n}}
$$

\section{Evalution of synthetic standard uncertainty}

The components are not related to each other and the combined uncertainty is calculated according to the square root.

$$
\begin{gathered}
u_{\text {crel }}(\omega)=\sqrt{u_{\text {rel }}^{2}(c)+u_{\text {rel }}^{2}(V)+u_{\text {rel }}^{2}(m)+u_{\text {rel }}^{2}(\text { rep })} \\
u_{c}(\omega)=u_{\text {crel }}(\omega) \times \omega
\end{gathered}
$$

$\mathrm{u}_{\text {crel }}(\omega)$ is $0.04,0.06$, respectively, $\mathrm{u}_{\mathrm{c}}(\omega)$ is $2.7,30 \mathrm{mg} / \mathrm{L}$, respectively.

\section{Evalution of extended uncertainty}

95\% confidence interval, including factor $\mathrm{K}=2$, $\mathrm{U}=5.4,60 \mathrm{mg} / \mathrm{L}$, respectively.

\section{Expression of analysis results}

The content of element Boron and Lead by ICP-OES is:

B : $\quad(66.3 \pm 5.4) \mathrm{mg} / \mathrm{kg}, \quad \mathrm{k}=2$ 
$\mathrm{Pb}: \quad(500 \pm 30) \mathrm{mg} / \mathrm{kg}, \quad \mathrm{k}=2$

\section{Conclustions}

In this paper, we established an uncertainty model for content of element $\mathrm{B}$ and $\mathrm{Pb}$ which can be used for evaluation of plastic pollution.

\section{References}

[1] Eriksen M, Maximenko N, Thiel M, et al. Plastic pollution in the South Pacific subtropical gyre. Marine Pollution Bulletin, 2013, 68(1-2):71-76.

[2] Azzarello M Y, Vleet E V. Marine birds and plastic pollution. Marine Ecology Progress, 1987, 37:295-303.

[3] Eriksen M, Lebreton LC, Carson HS, et al. Plastic Pollution in the World's Oceans: More than 5 Trillion Plastic Pieces Weighing over 250,000 Tons Afloat at Sea. Plos One, 2014, 9(12):e111913.

[4] Information on http://www.worldenvironmentday.global/en/about/beat-plastic-pollution

[5] Cai Zhongli, Li Xifeng. STUDIES ON THE PLASTIC POLLUTION ON THE SEA:A REVIEW. 1997.

[6] Yu X, Peng J, Wang J, et al. Occurrence of microplastics in the beach sand of the Chinese inner sea: the Bohai Sea Peng J, Wang J, et al. Occurrence of microplasti

[7] Yang Li-Li, Jiao Ze-peng, and Li Huan-Yong. Uncertainty Evaluation of Determination of Copper Content by ICP-AES. Chinese Journal of Spectroscopy Laboratory 1 (2013): 061.

[8] Hu Yang ,Li Huan-Yong, Jiao Ze-Peng, Hong Ai-Hua. Determination of Trace Metal Elements in Tea Saponin by ICP-AES with Microwave Digestion. Chinese Journal of Spectroscopy Laboratory 1 (2013): 056. 